\title{
The Interplay Between Spike-Time and Spike-Rate Modes in the Auditory Nerve Encodes Tone-In-Noise Threshold
}

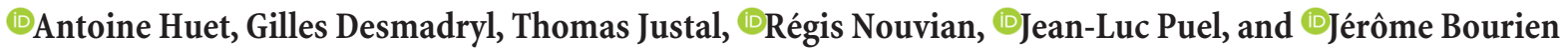 \\ INM, Inserm, Univ Montpellier, 34295 Montpellier, France
}

Auditory nerve fibers (ANFs) encode pure tones through two modes of coding, spike time and spike rate, depending on the tone frequency. In response to a low-frequency tone, ANF firing is phase locked to the sinusoidal waveform. Because time coding vanishes with an increase in the tone frequency, high-frequency tone coding relies on the spike rate of the ANFs. Adding a continuous broadband noise to a tone compresses the rate intensity function of ANFs and shifts its dynamic range toward higher intensities. Therefore, the ANFs with high-threshold/low-spontaneous rate (SR) are thought to contribute to behavioral tone detection in noise. However, this theory relies on the discharge rate of the ANFs. The direct comparison with the masking threshold through spike timing, irrespective of the spontaneous rate, has not so far been investigated. Taking advantage of a unique proxy to quantify the spike synchrony (i.e., the shuffle autocorrelogram), we show in female gerbils that high-SR ANFs are more adapted to encode low-frequency thresholds through temporal code, giving them a strong robustness in noise. By comparing behavioral thresholds measured using prepulse inhibition of the acoustical startle reflex with population thresholds calculated from ANFs pooled per octave band, we show that threshold-based spike timing provides a better estimate of behavioral thresholds in the low-frequency range, whereas the high-frequency behavioral thresholds rely on the spiking rate, particularly in noise. This emphasizes the complementarity of temporal and rate modes to code tone-in-noise thresholds over a large range of frequencies.

Key words: auditory nerve; noisy environments; rate coding; temporal coding

Significance Statement

There is a general agreement that high-threshold/low-spontaneous rate (SR) auditory nerve fibers (ANFs) are of prime importance for tone detection in noise. However, this theory is based on the discharge rate of the fibers. Comparing the behavioral thresholds and single ANF thresholds shows that this is only true in the high-frequency range of tone stimulations. In the low-frequency range of tones (up to $2.7 \mathrm{kHz}$ in the gerbil), the most sensitive ANFs (high-SR fibers) carry neural information through a spike-timing mode, even for noise in which tones do not induce a noticeable increment in the spike rate. This emphasizes the interplay between spike-time and spike-rate modes in the auditory nerve to encode tone-in-noise threshold over a large range of tone frequencies.

\section{Introduction}

Human hearing covers a large range of sound frequencies, from $\sim 20 \mathrm{~Hz}$ to $20 \mathrm{kHz}$. The pure-tone audiogram is the most common test used to assess the auditory threshold in experimental

Received Oct. 27, 2017; revised May 4, 2018; accepted May 10, 2018.

Author contributions: A.H., G.D., J.-L.P., and J.B. designed research; A.H., G.D., T.J., and J.B. performed research; A.H., R.N., J.-L.P., and J.B. contributed unpublished reagents/analytic tools; A.H., G.D., R.N., J.-L.P., and J.B. analyzed data; A.H., G.D., R.N., J.-L.P., and J.B. wrote the paper.

This work was supported by Inserm (Grant U1051-Dot 02-2015), Cochlear France (Grant R11055FF/ RVF11006FFA), Agence Nationale pour la Recherche (Grant ANR-13-JSV1-0009-01), and Prix Scientifique from Fondation pour l'Audition. We thank Scientific and Technical English Language Services (www.stels-ol.de) for editing assistance and laboratory members for valuable discussions and help, especially Hideaki Ogitta in the development of the startle response setup.

The authors declare no competing financial interests.

Correspondence should be addressed to Jérôme Bourien, INM, Inserm, Univ Montpellier, 80, rue Augustin Fliche, 34295 Montpellier Cedex 5, France. E-mail: jerome.bourien@inserm.fr.

DOI:10.1523/JNEUROSCI.3103-17.2018

Copyright $\odot 2018$ the authors $\quad 0270-6474 / 18 / 385727-12 \$ 15.00 / 0$ and clinical settings. In addition to external and middle ear transmission, the pure-tone audiogram relies on mechanotransduction followed by transforming the receptor potential of inner hair cells (IHCs) into action potential firing in auditory nerve fibers (ANFs). In response to a low-frequency tone burst, ANFs tuned at the tone frequency fire action potentials at a preferred phase of the sinusoidal waveform (i.e., neural phase locking). An examination of fiber response over a range of levels has shown that the appearance of neural phase locking may precede the elevation of the discharge rate above the fiber spontaneous rate (SR) (Rose et al., 1967; Anderson et al., 1971; Johnson, 1980). Above 2-3 kHz in mammals (for review, see Weiss and Rose, 1988), the receptor potential of IHCs cannot follow the fine structure of the tone because of the IHC's membrane time constant (Palmer and Russell, 1986). Neural phase locking progressively disappears and the fibers encode the stimulus envelope with a rate code; for tones, a steady elevation in rate that adapts with time after a peak at onset. 
When probing a range of tone levels, it was shown that an increment of the discharge rate above the fiber's SR precedes the advent of the neural onset response (Rhode and Smith, 1985). Altogether, these data suggest that spike timing and spike rate provide complementary neural cues for behavioral tone detection in quiet backgrounds.

When a tone burst is presented in addition to a continuous broadband noise, masking occurs because ANFs tuned to the tone frequency are driven by the noise. Investigations in animal models have shown that such masking produces a compression of the dynamic range and a shift of the rate intensity function toward higher tone levels (Costalupes et al., 1984). Increasing noise level leads toward a flat rate intensity function, with no detectable increment of discharge rate. Because this phenomenon affects the low-threshold/high-SR ANFs preferentially, low-SR fibers coding for higher levels are presumed to be more resistant to background noise (Costalupes et al., 1984; Young and Barta, 1986), making them of prime importance for detecting acoustic cues in a noisy environment through an increment in their discharge rate. Conversely, other studies support the idea that tone-innoise detection relies on temporal coding (Rhode et al., 1978; Abbas, 1981; Costalupes, 1985; Henry and Heinz, 2012). Phaselocked responses to low-level tones decrease with increasing masker level and generally remain reasonably strong above behavioral masking thresholds, suggesting that phase-locking mechanisms are involved in tone detection in noise. Finally, studies in humans suggest that tone detection in noise may be partly determined by neural phase locking to the fine structure of tone (Moore and Raab, 1975; Moore, 2003; Zeng et al., 2005; Lorenzi et al., 2006; Vinay and Moore, 2007; Kortlang et al., 2016). However, the respective weights of the spike-time and spike-rate mode in the auditory nerve in behavioral thresholds in noisy environments have never been fully examined in the same species. Moreover, it is still not known how ANFs adapt their encoding process in tone-in-noise; that is, do they keep their tone-in-quiet encoding modes (time vs rate) or do they switch from one to the other?

To address these questions, we designed an experimental protocol in gerbils to compare behavioral auditory threshold based on prepulse inhibition of the acoustical startle response (ASR) with physiological auditory thresholds based on single-fiber recordings in the auditory nerve. Here, we show that high-SR ANFs are more adapted to detect low-frequency tones in noise through temporal coding, whereas detection of high-frequency masked thresholds relies on rate coding through low-SR ANFs. This emphasizes the complementarity of spike time and spike rate to code tone-in-noise thresholds over a large range of frequencies.

\section{Materials and Methods}

Animals. Eighty female Mongolian gerbils ( $~ 3-4$ months old and weighing 51-60 g; behavioral experiments, $n=41$; electrophysiological experiments, $n=39$ ) were obtained from Janvier Laboratories. Housing and experimental procedures were approved by the French Ministry of Agriculture and Forestry (Housing Agreement C34-172-36; December 19, 2014; Experimental Agreements CEEA-LR-12111/CEEA-LR36). Experiments were performed in accordance with the animal welfare guidelines 2010/63/EC of the European Communities Council Directive regarding the care and use of animals for experimental procedures.

Prepulse inhibition of the ASR. Experiments were performed in a sound-attenuated booth (Kinder Scientific, SM 100SP-H) lined with acoustic foam panels to reduce acoustic reflections. Gerbils were placed in a custom, acoustically transparent cage that stood on a platform coupled to an accelerometer mounted at its base (Kinder Scientific, SM100MP-D). The acoustical stimuli were delivered by three distinct speakers (prepulse, Fostex FE 83 EN; pulse, Fostex FT17H; background noise, Tucker-Davis MF1, RRID:SCR_006495). Prepulse stimuli and background noise were generated by a custom-made LabVIEW (RRID: SCR_014325) software running on a PXI-4461 signal generator (National Instruments). Pulse generation and startle response acquisition were generated by startle monitor software (Kinder Scientific). Measurements were performed either in quiet or in the presence of $60 \mathrm{~dB}$ SPL continuous pink noise having an equal amount of energy per octave band (i.e., $1 / f$ noise, $\pm 3 \mathrm{~dB}$ fluctuations in octave bands within the range of $0.25-50 \mathrm{kHz})$. The pulse was a white noise burst of $50 \mathrm{~ms}$ duration $(1 \mathrm{~ms}$ rise and fall, $48 \mathrm{~ms}$ plateau) presented at $115 \mathrm{~dB}$ SPL. The prepulse was a tone burst of $50 \mathrm{~ms}$ duration ( $1 \mathrm{~ms}$ rise and fall, $48 \mathrm{~ms}$ plateau), varying in frequency $(0.5,1,2,4,8,16$, and $32 \mathrm{kHz})$ and in level $(0-50 \mathrm{~dB}$ SPL in $10 \mathrm{~dB}$ steps). The prepulse onset was positioned to start $100 \mathrm{~ms}$ before the pulse onset (Fig. 1A,B). Multiple sessions in random sequence (four in quiet and four in background noise) were performed on each animal to prevent fatigue and adaptation effects. For each background (quiet and noise), 20 presentations were used per frequency and level. The session lasted $\sim 1 \mathrm{~h}$ and animals were tested twice a day for 1 week (sessions separated by at least $6 \mathrm{~h}$ ). All session began with a $5 \mathrm{~min}$ acclimatization period before startle testing and the tests were conducted in lighted conditions. The intertrial interval varied randomly at $9 \pm 3 \mathrm{~s}$. The root mean square value of the ASR was calculated during the $100 \mathrm{~ms}$ after pulse onset (Fig. 1C,D).

Single-fiber recordings in the auditory nerve. Animals were anesthetized with an intraperitoneal injection of a mixture of $3 \mathrm{mg} / \mathrm{kg}$ xylazine (Rompun $2 \%$ ) and $40 \mathrm{mg} / \mathrm{kg}$ tiletamine/zolazepam (Zoletil 100) and placed in a head holder. Body temperature was maintained at $37.5 \pm 11^{\circ} \mathrm{C}$ using a thermostatic blanket (Harvard Apparatus). A posterior fossa approach was performed to expose the left cochlear nerve (for more details, see Bourien et al., 2014; Huet et al., 2016). Briefly, parts of the left occipital bone were removed, the left hemisphere of the cerebellum was aspirated, and the brainstem was displaced to expose the auditory nerve at the exit of the auditory meatus. Action potentials from single ANFs were recorded juxtacellularly using pulled (Sutter Instruments, P-1000) glass micropipettes (World Precision Instruments, 1B100 F-4) filled with $3 \mathrm{M}$ $\mathrm{NaCl}$ and connected to an amplifier (Molecular Devices, Axoclamp 2B). In vivo resistances were between 80 and $110 \mathrm{M} \Omega$. An $\mathrm{Ag}-\mathrm{AgCl}$ reference electrode was placed in the animal's neck.

Experiments were performed in a sound-attenuated booth. Calibrated acoustic stimuli were generated (National Instruments, PXI-4461) and delivered to the tympanic membrane using closed-field magnetic speakers (Tucker-Davis Technologies, MF1) coupled to ear bars. The signals recorded from individual ANFs were digitized using custom software (LabVIEW; National Instruments, RRID:SCR_014325) and saved for offline analysis (50 kilo samples/s, 24-bit analog-to-digital conversion resolution). The SR of each ANF was estimated from $30 \mathrm{~s}$ of activity in quiet and the characteristic frequency (CF) was determined using a custom-made, threshold-tracking program. Sound-driven activity was recorded in response to $50 \mathrm{~ms}$ tone bursts ( $1 \mathrm{~ms}$ rise and fall, 10 bursts/s) presented at the fiber CF. Sound-driven activity was recorded in quiet and in presence of a $60 \mathrm{~dB}$ SPL noise background shaped to be identical to the pink noise used for behavioral assessment (i.e., $1 / f$ noise, equal energy per octave band). A new noise stimulation was generated and used for each presentation by resetting the seed of a pseudorandom number generator on LabVIEW. The noise started at the same time as the tone, but the new noise (with new seed) begun at the sample immediately after the last noise had finished playing to create a continuous noise throughout the sound stimulation. Therefore, the tone and noise stimuli were not in-phase across the stimulus presentations. In total, 377 ANFs were recorded from 39 gerbils ( $\sim 10$ ANFs per animal). For each fiber, recording protocol duration took $\sim 20 \mathrm{~min}$, including SR and CF determination and intensity functions for tone-in-quiet and tone-in-noise stimulations.

Experimental design and statistical analysis. ASR intensity functions were recorded over a range of tone levels $(0-50 \mathrm{~dB}$ SPL in $10 \mathrm{~dB}$ steps, 20 presentations per level) and interpolated offline with $5 \mathrm{~dB}$ step linear interpolation. The behavioral audiogram was estimated for each frequency $(0.5,1,2,4,8,16$, and $32 \mathrm{kHz})$ across all the tested animals, using 
A

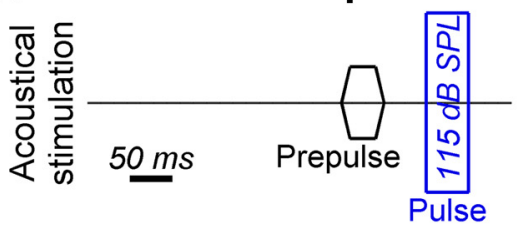

B

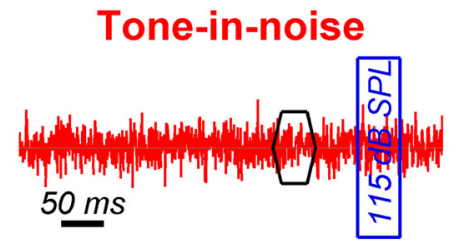

C
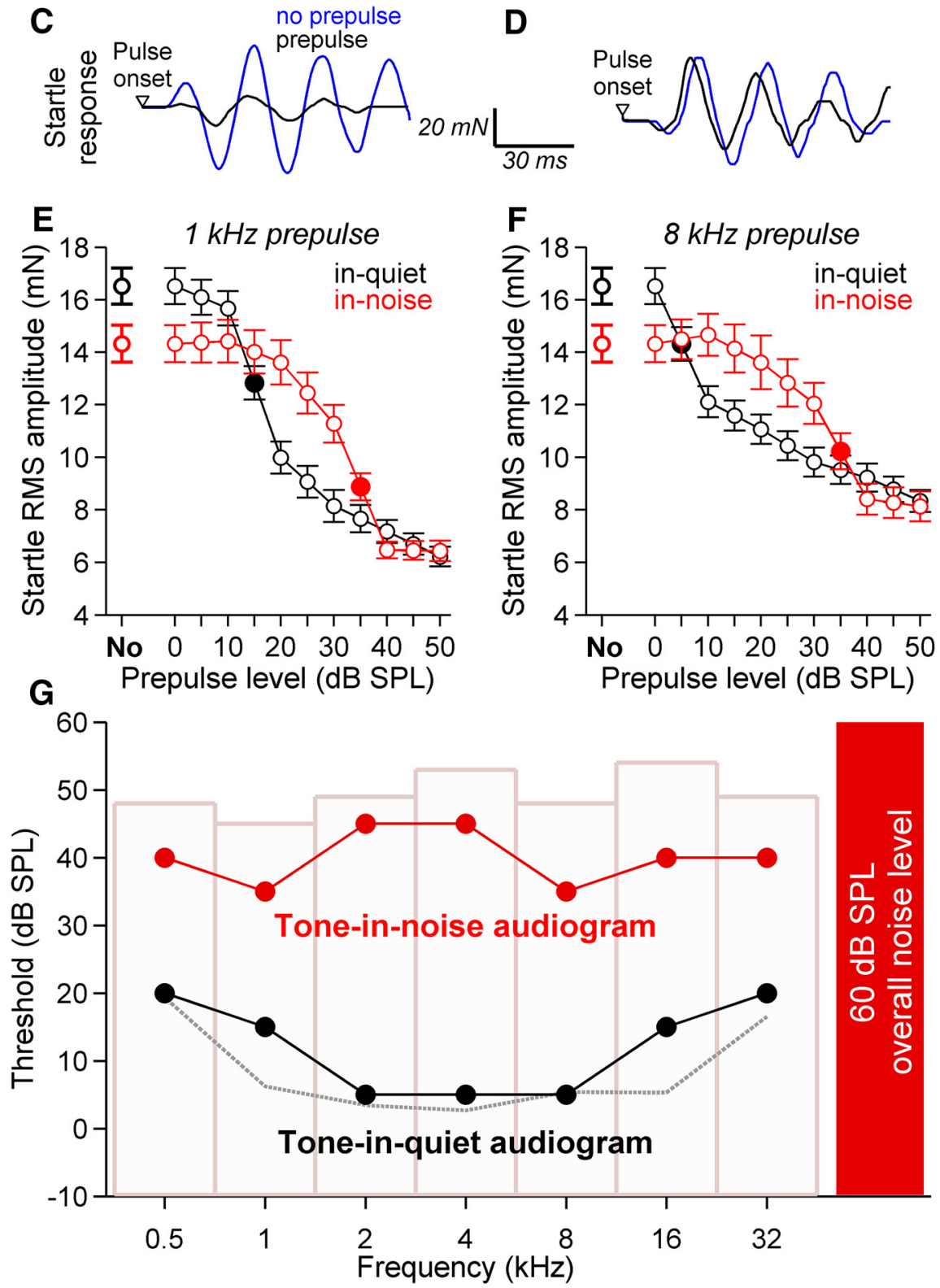

Figure 1. Behavioral assessment of gerbil auditory threshold in quiet and noisy backgrounds using prepulse inhibition of the ASR. $\boldsymbol{A}, \boldsymbol{B}$, Acoustic stimulation in quiet $(\boldsymbol{A})$ and background noise $(\boldsymbol{B})$. The pulse was a $50 \mathrm{~ms}$ white noise presented at $115 \mathrm{~dB}$ SPL. The prepulse, when it was present, was a $50 \mathrm{~ms}$ tone burst presented at a given level and frequency. Time interval between prepulse and pulse onsets was $100 \mathrm{~ms}$. $\boldsymbol{C}, \boldsymbol{D}$, Examples of startle reflex response in quiet $(\boldsymbol{C})$ and noise $(\boldsymbol{D})$ without (blue trace) or with (black trace) a $1 \mathrm{kHz}$ prepulse presented at $25 \mathrm{~dB}$ SPL. If the prepulse is detected by the auditory pathway (Fendt et al., 2001), then the startle reflex amplitude is reduced compared with the no-prepulse condition. $\boldsymbol{E}, \boldsymbol{F}$, Startle amplitude expressed in mN RMS as a function of prepulse level is shown for $1 \mathrm{kHz}(\boldsymbol{E})$ and $8 \mathrm{kHz}(\boldsymbol{F})$ prepulses presented in quiet (black) or noise (red) in 41 gerbils. Closed symbols show the level for which a significant inhibition of the startle amplitude was detected. G, Behavioral audiograms in quiet (black trace) and in noise (red trace). The pink histogram represents the spectrum of the noise per octave band. The solid red bar on the right side of the panel is the overall noise level. The gray dashed curve is the gerbil tone-in-quiet audiogram reported in Ryan (1976). a $d^{\prime}$ statistical test (Macmillan and Creelman, 2004) with the no prepulse condition as the reference. The lowest tone level with $d^{\prime} \geq 1$ was defined as the behavioral threshold.

Quantification of the spike synchrony was performed using the shuffle autocorrelogram and its derived correlation index (CI) (Louage et al., 2004; Joris et al., 2006) during the tone burst duration and across the 60 presentations at the same sound level. The CI corresponds to the height of the central peak of the normalized shuffled autocorrelogram (SAC). The CI varied from 1 for random distribution of spikes to a large value when spikes fired in synchrony. Rate and $\mathrm{CI}$ intensity functions were recorded over a range of stimulus levels $(0-80 \mathrm{~dB}$ SPL in $4 \mathrm{~dB}$ steps, 60 presentations per level, presented in random order of levels) and interpolated offline using $1 \mathrm{~dB}$ step linear interpolation. The rate-based threshold of each fiber was determined using a $d^{\prime}$ statistical test with the $0 \mathrm{~dB}$ SPL condition as the reference (Macmillan and Creelman, 2004). The lowest tone level with $d^{\prime}$ $\geq 1$ was defined as the rate-based threshold. The CI-based threshold of each fiber was determined using a bootstrap method by simulating "Poisson spike trains" having the same spike rate statistics as experimental spike trains. A statistical threshold was calculated for $p<0.05$ over 200 scrambled versions of each spike train (Joris et al., 2006). The lowest tone level that elicited a significant CI value defined the CIbased threshold. To estimate the rate- and CIbased population thresholds, ANFs were pooled per octave band (from $500 \mathrm{~Hz}$ to 32 $\mathrm{kHz}, 1$ octave increments) and a one-way ANOVA test was applied to rate and CI intensity functions. The level at which a significant increment of the function populations was detected defined the population threshold ( $p<$ 0.05 , Tukey's post hoc test). Data are expressed as the mean \pm SEM. Analysis was performed using MATLAB (RRID:SCR_001622) software and its statistics and signal-processing toolboxes.

\section{Results}

All animals used in this study showed a Preyer's reflex in response to finger snaps. The reflex was considered as positive when a rapid pinna movement was clearly noticed by visual inspection. Prepulse inhibition of the ASR was performed to measure behavioral auditory thresholds in quiet and in background noise $(n=41$; Fig. $1 A, B)$. In another pool of gerbils $(n=$ 39 ), the single-unit activity in the auditory nerve was recorded under the same paradigm of quiet and background noise, allowing the correlation of behavioral and physiological thresholds. The background noise was presented continuously at an overall level of $60 \mathrm{~dB}$ SPL and corresponded to a pink noise (i.e., $1 / f$ noise) characterized by an equal amount of energy per octave band (see vertical red bar plots in Fig. 1G). 


\section{Behavioral audiogram}

As suggested previously, the prepulse inhibition of the ASR is a reliable proxy for the tone audiogram in quiet and noisy environments (Hickox and Liberman, 2014). In a quiet background, a prepulse tone burst at 1 or $8 \mathrm{kHz}$ can modulate the startle response amplitude at sound pressures as low as 15 or $5 \mathrm{~dB}$ SPL, respectively (Fig. 1E,F). Adding $60 \mathrm{~dB}$ SPL background noise induced a rightward shift in the startle response intensity functions in noise compared with those measured in quiet, leading to a 20 and $30 \mathrm{~dB}$ threshold elevation at 1 and $8 \mathrm{kHz}$, respectively (Fig. 1E, F, red curves). A slight but significant reduction in the startle response amplitude was observed in the presence of the background noise, which could reflect an adaptation of this auditory motor reflex to sustained noise stimulation (Davis, 1974). Based on this method, we determined a behavioral audiogram for the tone burst prepulse, varying from $0.5-32 \mathrm{kHz}$ in 1-octave increments (Fig. 1G). In quiet, the audiogram displayed a $\mathrm{U}$ shape, similar to that obtained with an operant procedure (Ryan, 1976). Although the energy of the background noise was relatively flat per octave band, the behavioral threshold shift in background noise varied from $20-40 \mathrm{~dB}$. The maximum threshold shift $(+40 \mathrm{~dB})$ was observed at 2 and $4 \mathrm{kHz}$ (Fig. 1G). Furthermore, the tone-in-noise audiogram remained below the noise overall level (Fig. 1G), indicating the robust capability of gerbils to detect tone stimulation in noisy environments (Costalupes, 1983; Kittel et al., 2002).

\section{ANF recordings}

Using single-fiber recordings in the auditory nerve, we determined the ANF's CF (i.e., the frequency for which the fiber is the most sensitive) and its SR over $30 \mathrm{~s}$. We then recorded the same units in response to a $50 \mathrm{~ms}$ tone burst (10/s) presented at CF in quiet and in the presence of continuous noise. Figure 2 shows two representative examples of high-SR ANFs, with a CF close to 1 and $8 \mathrm{kHz}$ (Fig. 2A-D). Plotting raster plots and corresponding peristimulus time histograms showed that adding noise decreased the overall spike rate during the tone-burst; that is, the peak and plateau were reduced (Fig. $2 E-L$ ). To determine the degree of spike synchronization across presentations of the stimulus, we measured the CI, which corresponds to the central peak amplitude of the normalized SAC (Fig. 2I-L, insets). For the low-CF ANFs, the SAC is characterized by a large central peak (even in presence of noise) and multiple adjacent oscillations, attesting to strong neural phase locking to the fine structure of the tone burst. For the high-CF ANFs, the peak height of SAC and adjacent oscillations were strongly reduced, indicating that neural phase locking failed (Fig. $2 K, L$, insets). In this fiber, the remaining central peak mostly reflected the small temporal jitter of the first spike at the tone burst onset.

We then plotted $\mathrm{CI}$ and rate intensity functions to determine the ANF thresholds in quiet and noise (Fig. $2 M-P$ ). The difference between time- and rate-based thresholds ( $\Delta$ threshold) was used to characterize the most sensitive metrics between both (see horizontal arrows in Fig. $2 M-P$ ). Negative or positive $\Delta$ threshold values indicate that the lowest thresholds are achieved in the time or rate domain, respectively. High-SR/low-CF ANFs displayed a preferential coding in the time domain (Fig. $2 M, N$ ) independently of the presence of background noise [change in threshold $(\Delta \mathrm{th})=-8$ and $-16 \mathrm{~dB}$ in quiet and noise, respectively]. However, a low-SR/low-CF ANF that displayed positive $\Delta$ threshold in quiet ( $\Delta$ th $=+4 \mathrm{~dB}$ ) became negative in background noise ( $\Delta$ th $=-12 \mathrm{~dB}$; Fig. $2 Q, R)$, indicating a change in encoding mode from spike rate in quiet to spike time in noise. Finally, low-SR/high-CF ANF (Fig. 2O,P) had the same behavior as the high SR/high CF (Fig. 2S,T): that is, they used spike rate (positive $\Delta$ threshold) to code threshold in both quiet and noisy environments.

We then applied this analysis to 377 ANFs (CFs ranged from $0.3-45 \mathrm{kHz}$, median value $5.7 \mathrm{kHz}$; SRs ranged from $0.03-130$ spikes/s, median value 26 spikes/s). For each fiber, the CI-based threshold was plotted as a function of its rate-based threshold. In quiet (Fig. $3 A$ ), most of the ANFs were distributed on both sides of the $y=x$ line, indicating that the lowest ANF thresholds can be obtained by either using the time-based analysis (below the $y=x$ line) or the rate-based analysis (above the $y=x$ line). Lower-CF fibers (Fig. 3A, purple dots) displayed lower thresholds when calculated from CI, whereas the threshold of higher-CF ANFs (Fig. 3A, orange dots) was lower when derived from discharge rate estimates, attesting to a CF dependency of the mode of coding. To confirm the frequency dependence of the mode of coding (rate vs time), the $\Delta$ threshold of each fiber was plotted against the corresponding CF (Fig. 3B). Except for a small fraction of low-SR units that are located just above the $x$-axis, the majority of the lowest-CF fibers displayed a negative $\Delta$ threshold (Fig. $3 B, C$ ), suggesting that these fibers fire synchronized action potentials across acoustic trials without elevating their discharge rate. In contrast, the highest-CF show a positive $\Delta$ threshold, suggesting that these fibers are able to increase their spiking rate without a synchronized pattern of action potential firing. Interestingly, fitting of this distribution with a sigmoid indicated a cutoff frequency between the two modes of coding of $\sim 2.7 \mathrm{kHz}$ (Fig. $3 B$ ), consistent with the roll-off value of neural phase locking in gerbil auditory nerve (Versteegh et al., 2011).

Next, we expressed $\Delta$ threshold as a function of the fiber SR (Fig. 3D). All of the low-SR ANFs showed a positive $\Delta$ threshold in quiet, indicating that spike-rate analysis resulted in a better threshold estimates. This result can be most likely explained as low-SR ANFs having to elevate first their firing rate above their SR before organizing their action potentials in synchrony with the sound stimulation. Beyond 20 spikes/s, the high-CF ANFs still displayed a positive $\Delta$ threshold, although a small reduction in $\Delta$ threshold amplitude was observed (Fig. $3 D, E$ ). This reduction in $\Delta$ threshold can be explained by the smaller jitter of the high-SR relative to low-SR units, leading to a more robust CI-based threshold (Huet et al., 2016). The cutoff value at 20 spikes/s is likely related to the duration of the tone burst used in our paradigm (i.e., $50 \mathrm{~ms}$ ). Indeed, a minimum of one spike per burst is required to operate phase-locking; that is, to fire in synchrony across trials. In others words, if a tone burst duration had been set to 25 or $100 \mathrm{~ms}$, then the cutoff value would be estimate at 40 or 10 spikes/s, respectively.

Adding $60 \mathrm{~dB}$ SPL background noise led to a general threshold elevation, reflected by the large deviation of the CI- and ratebased threshold coordinates in respect to the $y=x$ line (Fig. $3 F$ ). Time- and/or rate-based threshold could not be detected in a notable number of ANFs because their CI and/or rate intensity functions were almost flat. For $19 \%$ of ANFs, the CI and rate intensity functions were both strongly compressed, making it impossible to assign any threshold (Fig. 3F, $\mathbf{\square}$ ). Most of these fibers populate the basal part of the cochlea (CF median value of $9 \mathrm{kHz}$; SR median value of 22 spikes/s). Eleven percentage of ANFs without a measurable CI-based threshold, but still having a rate-based threshold (Fig. $3 F, \mathbf{\Delta}$ ) originated in the base of the cochlea (CF median value of $8 \mathrm{kHz}$; SR median value of 10 spikes/ s). In contrast, the $22 \%$ of ANFs without a measurable rate-based threshold, but having a CI-based threshold (Fig. 3F, $>$ ) were 


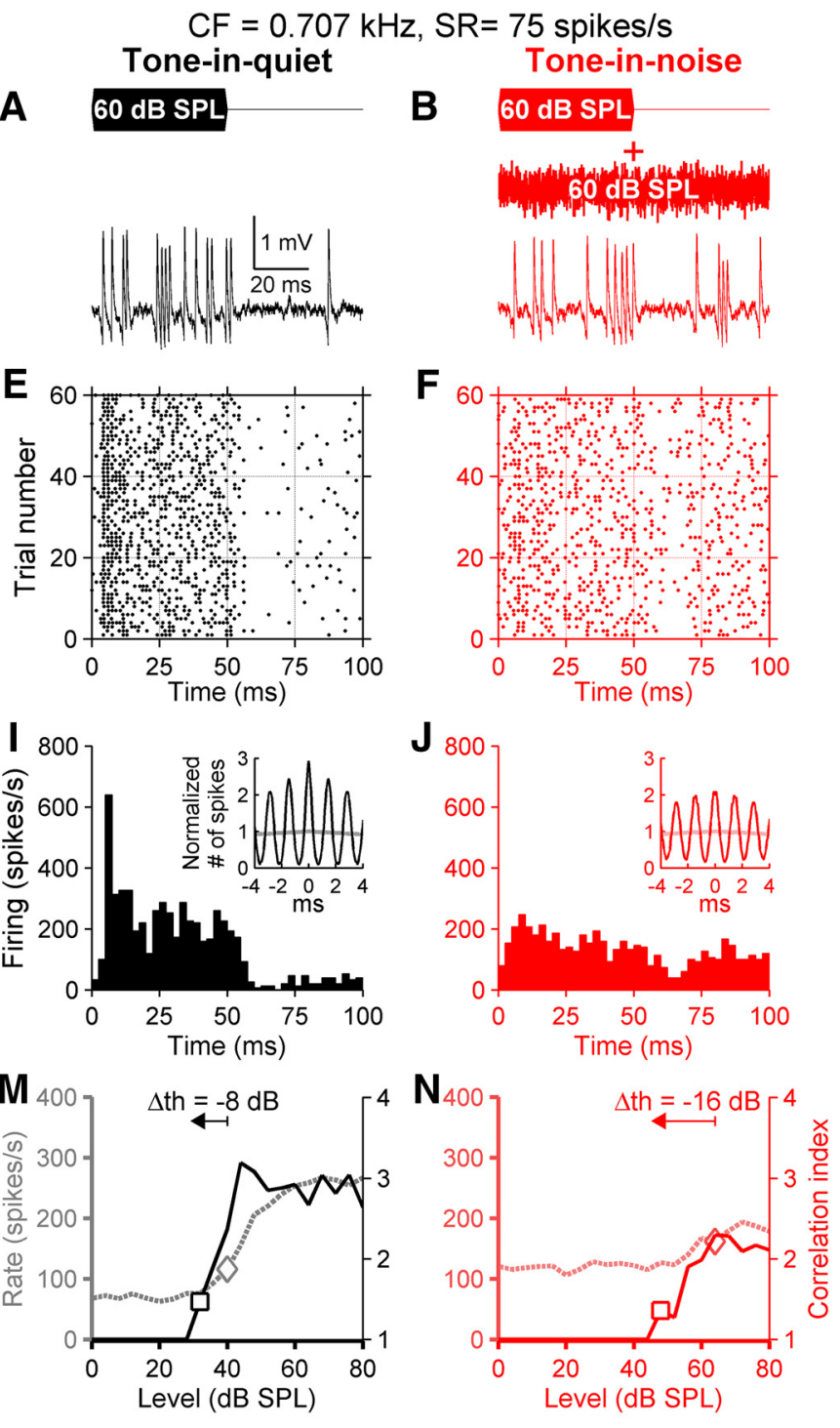

$\mathrm{CF}=0.943 \mathrm{kHz}, \mathrm{SR}=0.7$ spike/s
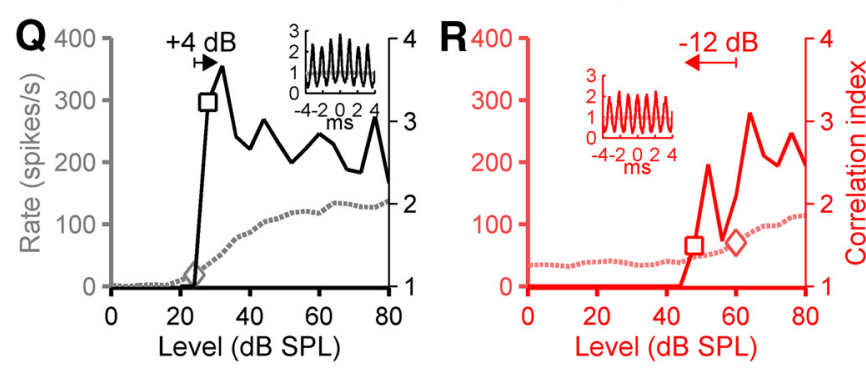
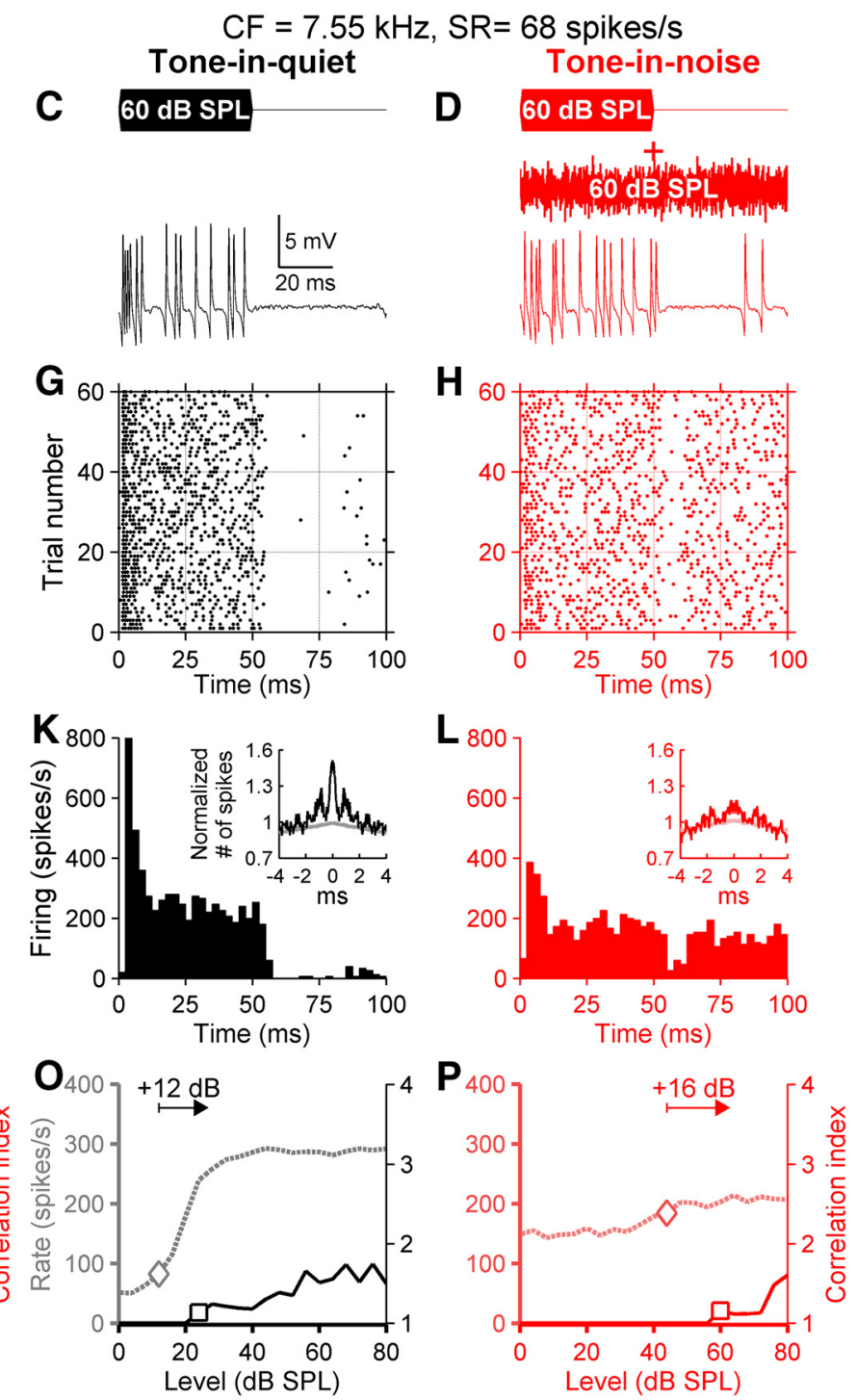

$\mathrm{CF}=7.127 \mathrm{kHz}, \mathrm{SR}=0.8$ spike/s
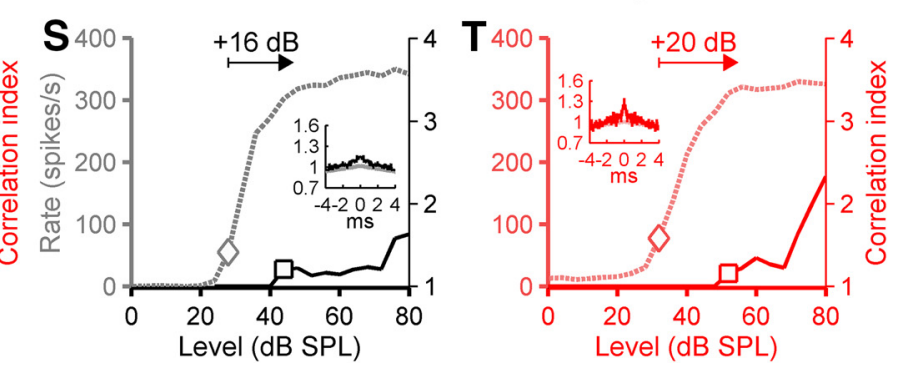

Figure 2. Tone burst responses of auditory nerve fibers in quiet and noise. $\boldsymbol{A}-\boldsymbol{D}$, Response of a low-CF/high-SR fiber $(\boldsymbol{A}, \boldsymbol{B})$ and a high-CF/high-SR fiber $(\boldsymbol{C}, \boldsymbol{D})$ to a $60 \mathrm{~dB}$ SPL tone burst presented at the fiber (F, in quiet (black) and in noise (red). $\boldsymbol{E}-\boldsymbol{H}$, Dot raster plots showing spike times for the fibers shown above to multiple presentations of the stimulus. I, $\boldsymbol{J}$, Peristimulus time histograms (binwidth $=2.5 \mathrm{~ms}$ ) computed from the panels shown above. Insets display the normalized shuffled autocorrelograms (binwidth $=60 \mu \mathrm{s}$ ) computed from the panels shown above. The light-colored curve presents the mean SAC calculated from 200 bootstrap resampling of data using "Poisson spike trains" having the same spike rate statistics as experimental spike trains. The Cl was defined as the amplitude of the central peak of the SAC (Joris et al., 2006). $\boldsymbol{M}-\boldsymbol{P}$, Rate intensity (dashed curve, left axis) and Cl intensity functions (solid curve, right axis) in quiet ( $\boldsymbol{M}, \mathbf{0}$ ) and noisy $(\boldsymbol{N}, \boldsymbol{P})$ backgrounds for the two fibers shown above. The symbols indicate the threshold for which a significant increment of the response was detected using statistical analysis of the neural response (rate intensity function, open diamond; $\mathrm{Cl}$ intensity function, open square). $\Delta$ th indicates the difference between $\mathrm{Cl}$ - and rate-based thresholds reference rate. $\mathbf{Q}-\boldsymbol{T}$, Rate and $\mathrm{Cl}$ intensity functions in quiet and in noise for two fibers with low SR. Insets are normalized SACs in response to a $60 \mathrm{~dB}$ SPL tone burst. For two high-CF fibers, the small but significant remaining SAC central peak reflects the synchronicity of the first spike evoked by a tone at the beginning of the burst. Note that $\mathrm{Cl}$ intensity function of low-CF fibers reaches a maximum value $20 \mathrm{~dB}$ above threshold and declines progressively for higher levels $(\boldsymbol{M}, \boldsymbol{N}, \mathbf{Q}, \boldsymbol{R})$, as shown previously with the measurements derived from period histogram (Johnson, 1980). In contrast, the Cl intensity function of high-CF fibers growths monotonically with level above threshold $(\boldsymbol{O}, \boldsymbol{P}, \boldsymbol{S}, \boldsymbol{T})$. 


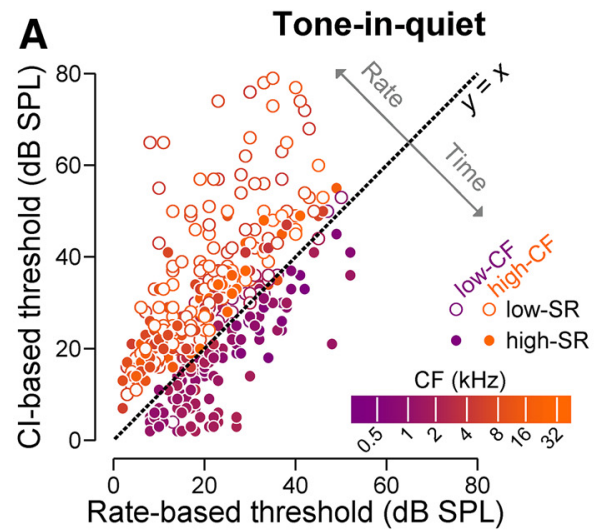

B
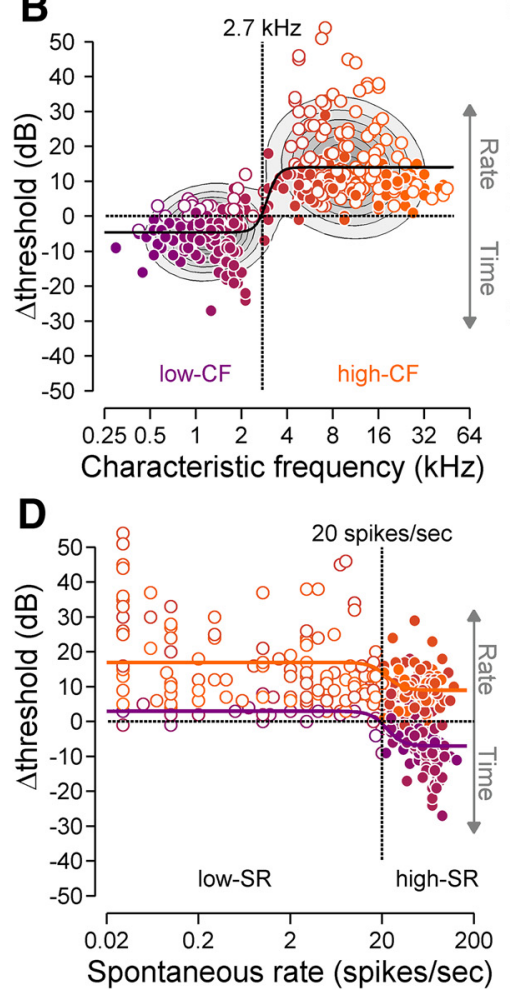

C
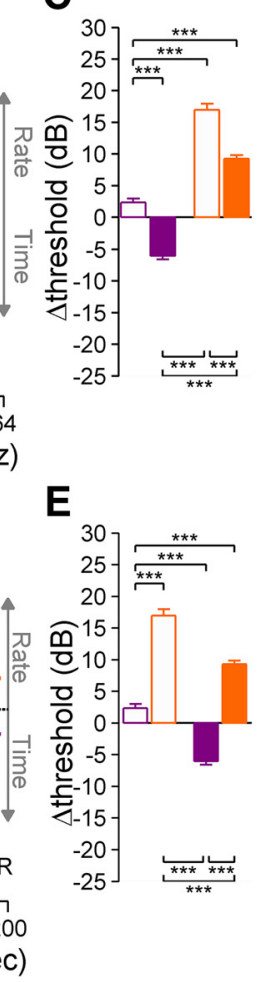

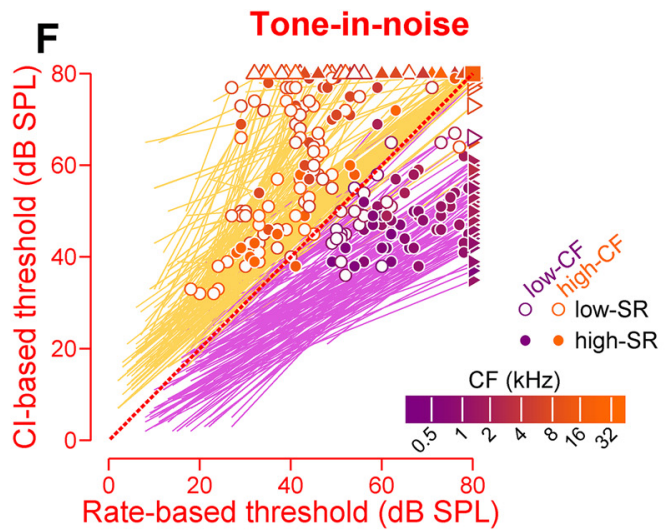

G

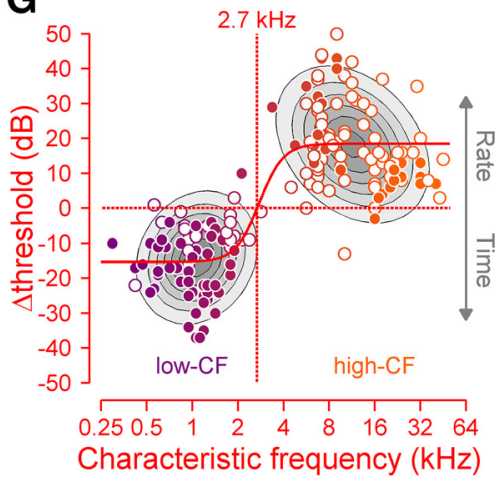

H

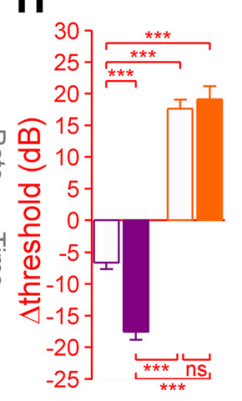

I

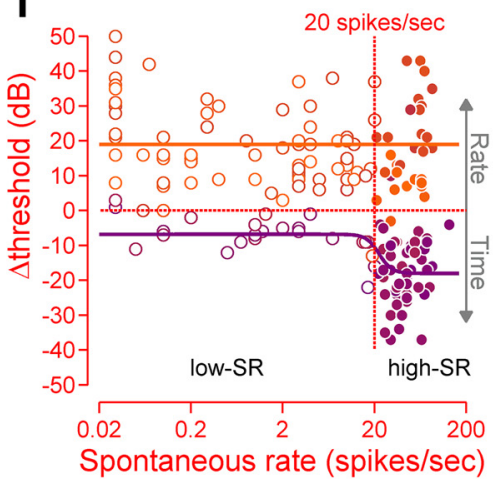

$\mathbf{J}$

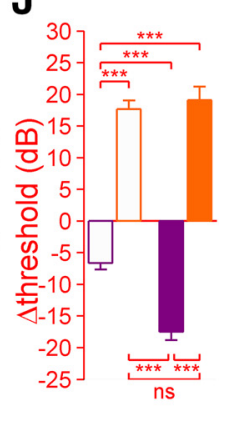

Figure 3. Sensitivity of ANFs estimated from spike time or spike rate depends of CF and SR.A, $F$, For each fiber ( $n=377)$, its (I-based threshold was plotted as a function of its rate-based threshold in quiet $(\boldsymbol{A})$ and noisy $(\boldsymbol{F})$ conditions. A color map was used to indicate the fiber $C F$ (from purple for lowest $C F$ to orange for highest $C F$ ). Light-colored thin lines in $\boldsymbol{F}$ indicate the threshold shift induced by noise. Open and closed symbols were used to indicate low-SR fibers $(<20$ spikes/s) and high-SR fibers, respectively. In $\boldsymbol{F}$, three different symbols $(\boldsymbol{\Lambda}, \boldsymbol{\nabla}, \mathbf{\square})$ were used to show fibers for which no measurable increment in the response was observed. $B, G$, Difference between the rate-based and CI-based thresholds ( $\Delta$ threshold) as a function of the fiber $(F$ from data shown above. Positive values indicate that the rate-based threshold is below the Cl-based threshold and negative values indicate the opposite. Data were fitted with sigmoidal models. Vertical dashed lines demarcate the sign change in fitting models as a proxy for the cutoff frequency value (i.e., $2.7 \mathrm{kHz}$ ). This value is confirmed by fitting of data with a mixture of bivariate Gaussian distributions (see isoprobability contours in gray). $\boldsymbol{D}, \boldsymbol{I}, \Delta$ threshold as a function of the fiber $S R$ derived from data shown in $\boldsymbol{A}$ and $\boldsymbol{F}$, respectively. Data were fitted with sigmoidal models. Vertical dashed lines demarcate the sign change in fitting model as a proxy of the cutoffSR value (i.e., 20 spikes/s). $\boldsymbol{C}, \boldsymbol{E}, \boldsymbol{H}, \boldsymbol{I}$, Quantification of data shown in $\boldsymbol{B}, \boldsymbol{D}, \mathbf{G}, \boldsymbol{I}$, respectively. ${ }^{* * *} p<0.001 ; \mathrm{ns}, p>0.1$, Student's $t$ test. In $\mathbf{G}-\boldsymbol{J}$, fibers with no detectable rate-based and/or Cl-based thresholds were not taken into account in the analysis.

distributed in the cochlear apex and displayed a high SR (CF median value of $1.7 \mathrm{kHz}$; SR median value of 50 spikes/s). The remaining fibers amounted to $46 \%$ and exhibited measurable time and rate thresholds (Fig. $3 F, \mathbf{O}$ ).

Plotting the $\Delta$ threshold of the remaining $46 \%$ fibers as a function of their CF indicates that they originate from apical and basal parts of the cochlea (CF median value of $6.7 \mathrm{kHz}$; SR median value of 16 spikes/s; Fig. 3G). The sensitivity of low-CF ANFs mostly relied on temporal cues, whereas the high-CF exhibited a better sensitivity when analyzed through the rate (Fig. $3 G, H$ ). The comparison of sigmoidal fits and bivariate Gaussian distributions shows that background noise tends to exaggerate this time versus rate specialization without changing the cutoff frequency, which remains at $2.7 \mathrm{kHz}$ (Fig. $3 \mathrm{~B}, \mathrm{G}$ ). In addition, the $\Delta$ threshold value of high-CF ANFs is completely independent of their corresponding SR with background noise (Fig. $3 I, J$ ). The increase in the discharge rate in the high-SR/high-CF fibers induced by the noise may increase the first spike jitter, leading to a weaker CI-based threshold and therefore to a more robust ratebased threshold. Conversely, the $\Delta$ threshold amplitude of low-CF ANFs remains dependent on SR (Fig. 3I,J). As also shown in Figure 2, $Q$ and $R$, a small number of fibers corresponding to low-SR ANFs from the apex switched from rate coding in quiet to temporal coding in noise (Fig. $3 D, I$ ) because noise in- 

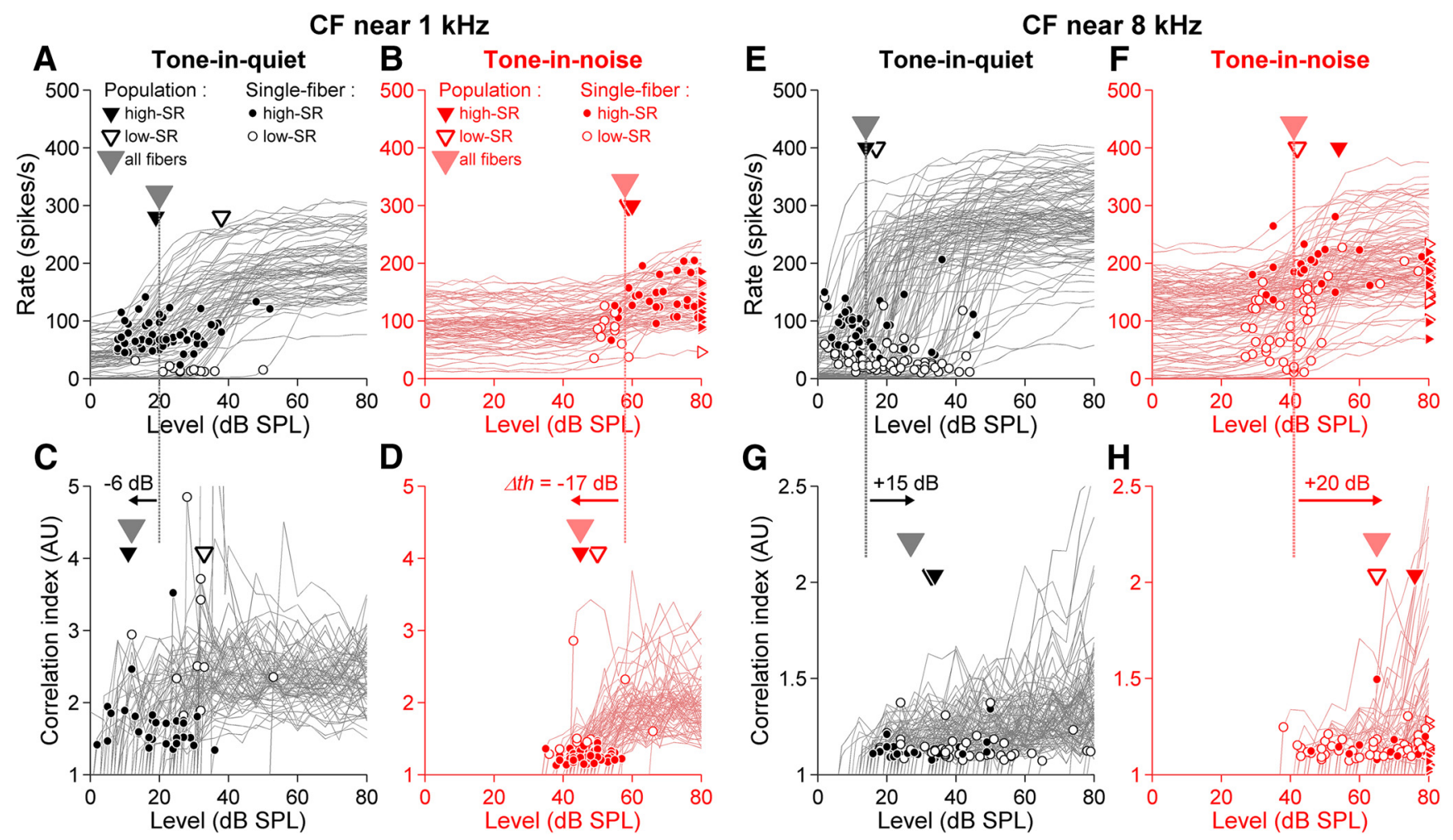

Figure 4. Single-fiber and population thresholds in response of tone-in-quiet (black axes) and tone-in-noise (red axes) for fibers with a CF located in the octave band centered on $1 \mathrm{kHz}$ ( $\boldsymbol{A}-\boldsymbol{D}$ ) and $8 \mathrm{kHz}(\boldsymbol{E}-\boldsymbol{H})$. Thin curves are rate intensity functions (top row) and Cl intensity functions (bottom row) of single fibers. Small symbols indicate individual fiber threshold distinguishing low-SR fibers $(<20$ spikes/s, open circles) from high-SR fibers (closed circles). Large symbols indicate the population's threshold estimated with a one-way ANOVA test using the level as the factor, considering all of the fibers (gray triangle symbols) or separating low-SR ( $<20$ spikes/s, open triangles) from high-SR fibers (closed triangles). $\Delta$ th indicates the difference between $\mathrm{Cl}$ - and rate-based population thresholds calculated from the whole population of ANFs. Small triangle symbols in $\boldsymbol{B}$ and $\boldsymbol{F}$ were used when no measurable increment in the response of the fiber could be detected.

duces enough spikes for the low-SR ANFs to response in phase locking. Interestingly, some low-CF/high-SR ANFs were able to phase lock $30 \mathrm{~dB}$ below the level that induced a detectable elevation of the discharge rate (Fig. $3 G, I$ ).

The above analysis relies on individual fibers, which still respond to tone-in-noise, but excluding fibers without noticeable CI- or rate-based threshold because the CI and rate intensity functions were almost flat. However, a small increment in the intensity functions may become significant when combined across ANFs innervating the same cochlear region. Therefore, we pooled the ANFs per CF octave band to determine the population threshold. Rate- and CI-based population thresholds were estimated using a one-way ANOVA test with the tone level as parameter. Figure 4 shows the population threshold for fibers with CFs near 1 or $8 \mathrm{kHz}$. For the $1 \mathrm{kHz}$ range, a lowest threshold was found when calculated through CI rather discharge rate mode, in quiet and in background noise (Fig. $4 A-D$ ). This result is consistent with the neural phase-locking response in this low range of stimulation, but contrasts with the analysis based on individual threshold, in which a small fraction of fibers with low CF/low SR have been found to have the lowest rate-based threshold (Figs. $2 Q, 3 F)$. However, the small size of this fiber pool (6\%) makes it insignificant at the population level. For $8 \mathrm{kHz}$, the lowest threshold is observed when calculated with the rate-based analysis both in quiet and in background noise (Fig. $4 E, F$ ). We then distinguished the population's threshold according to the fiber's SR. For tone-in-quiet, the whole population threshold overlaps with those from high-SR ANFs (Fig. $4 A, C, E, G$ ). In response to tonein-noise, the population threshold fits rather well with the threshold distribution of low-SR ANFs (Fig. $4 F, H$ ) except for the
$1 \mathrm{kHz}$ range, in which the population threshold of low-SR and high-SR ANFs are rather similar (Fig. 4B,D). As already pointed out for the individual fibers shown in Figure 2, $M-T$, the neural population threshold at $1 \mathrm{kHz}$ estimated from spike timing is better than the threshold based on rate $(\Delta \mathrm{th}=-6$ and $-17 \mathrm{~dB}$ in quiet and noise, respectively; Fig. $4 C, D)$. At $8 \mathrm{kHz}$, the threshold was lowest when approximated through the rate-based analysis ( $\Delta$ th $=+15$ and $+20 \mathrm{~dB}$ in quiet and noise, respectively; Fig. $4 G, H)$.

\section{Spike rate and spike time predict the behavioral audiogram}

To determine which neural cues (spike time or spike rate) provide the best prediction of auditory threshold, we compared the behavioral thresholds measured with the prepulse inhibition of the ASR with the ANF population thresholds (Fig. 5). In quiet, rate-based neural thresholds overestimate the behavioral audiogram of $11.4 \pm 2.4 \mathrm{~dB}$ with no significant difference between low-CF $(<2.7 \mathrm{kHz})$ and high-CF $(>2.7 \mathrm{kHz})$ ANFs (Fig. $5 A, E)$. In the presence of background noise, an elevation of behavioral and neural thresholds was observed (threshold difference $=$ $15.7 \pm 4.5 \mathrm{~dB}$ on all the frequencies), but in a strongly frequencydependent manner for the neural threshold (Fig. $5 B, D$ ). Whereas a rate-based threshold fits the behavioral threshold $>2.7 \mathrm{kHz}$ $(7.7 \pm 4 \mathrm{~dB})$, it fails to follow the low-frequency behavioral threshold and overestimates these thresholds by $26 \pm 2.8 \mathrm{~dB}$ (Fig. $5 B, F)$. In contrast, the neural threshold estimated from CI corresponded well to the low-frequency behavioral audiogram (up to $2.7 \mathrm{kHz})$ in quiet and noise $(5.3 \pm 4.2 \mathrm{~dB}$ and $7.7 \pm 1.5 \mathrm{~dB}$ difference in quiet and noise, respectively), but did not match with the behavioral thresholds in the high-frequency range (19 \pm 
A
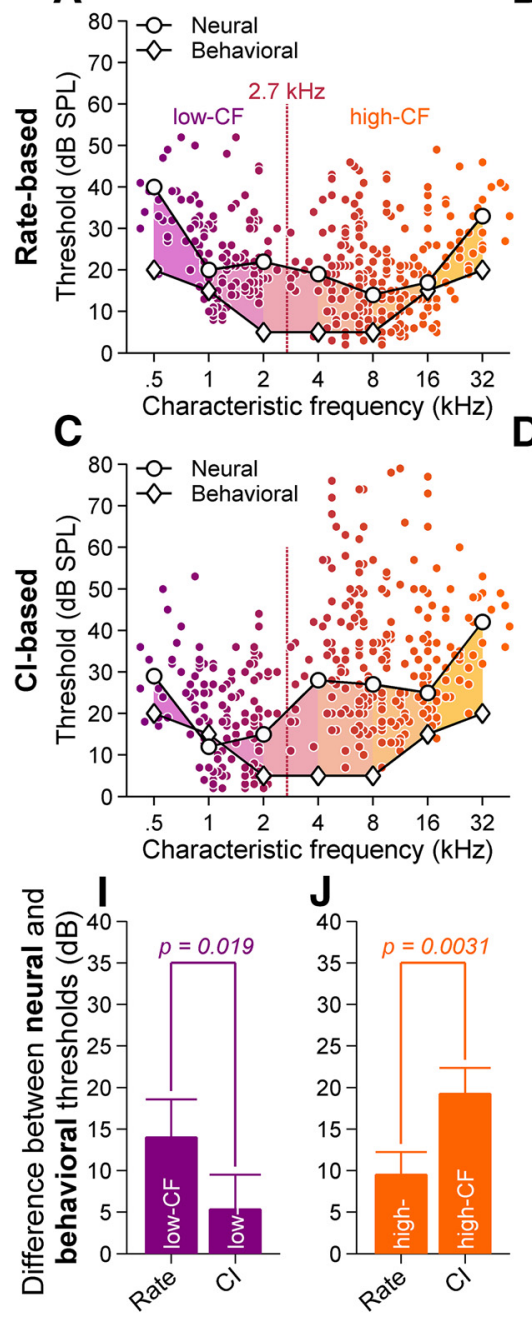

J

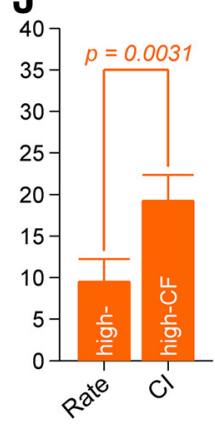

B
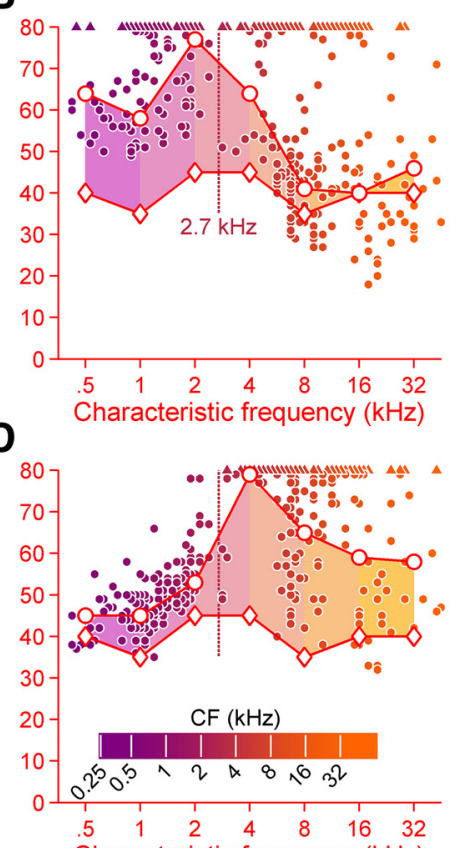

Characteristic frequency $(\mathrm{kHz})$
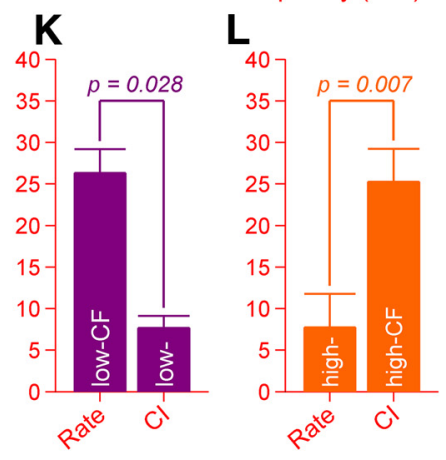

$\mathbf{E}^{\text {Tone-in-quiet }} \mathbf{F}^{\text {Tone-in-noise }}$
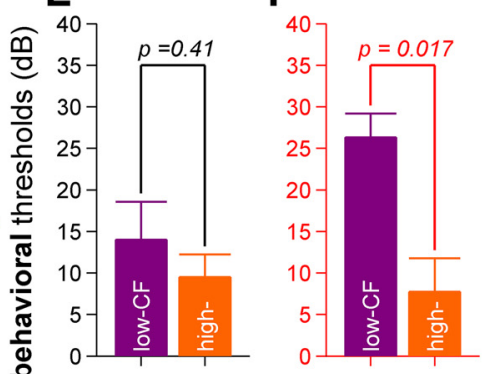

\section{H}
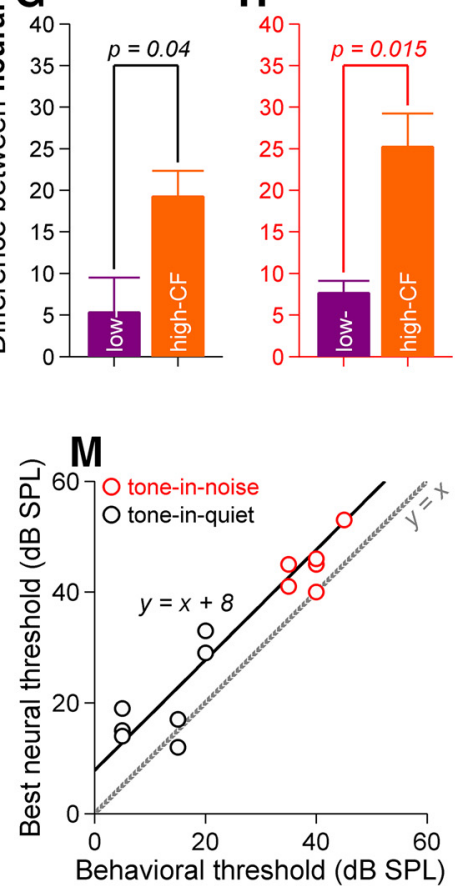

Figure 5. Neural sensitivity of ANFs and behavioral auditory threshold in quiet and noisy backgrounds. $\boldsymbol{A}, \boldsymbol{B}$, Rate-based population threshold (large open circles) and behavioral threshold (large open diamonds) as a function of $(F$ in quiet $(\boldsymbol{A})$ and noisy $(\boldsymbol{B})$ conditions. Note the gap in the low-frequency region between the tone-in-noise behavioral audiogram and the population thresholds of ANFs (filled area in $\boldsymbol{B}$ ). $\boldsymbol{C}, \boldsymbol{D}$, Cl-based population threshold (large open circles) and behavioral threshold (large open diamonds) as a function of (F in quiet ( $\boldsymbol{C}$ ) and noisy ( $\boldsymbol{D}$ ) conditions. In $\boldsymbol{B}$ and $\boldsymbol{D}$, a symbol ( $\mathbf{\Delta})$ indicates fibers with no detectable threshold $(\boldsymbol{B}, n=156 ; \boldsymbol{D}, n=113 \mathrm{ANFs})$, small circle symbols indicate single-fiber's threshold. $\boldsymbol{E}$ - $\boldsymbol{H}$, Quantification of the data shown in the $\boldsymbol{A}-\boldsymbol{D}$, respectively, separating low-CF fibers ( $<2.7 \mathrm{kHz}$, purple histograms) and high-CF fibers ( $\geq 2.7 \mathrm{kHz}$, orange histograms). $\boldsymbol{E}-\boldsymbol{L}$, Quantification of the data shown in the $\boldsymbol{A}-\boldsymbol{D}$, considering population thresholds derived from rate- and Cl-based analysis. Purple and orange histograms show the difference (mean \pm SEM) between neural and behavioral thresholds in low-frequency ranges ( $<2.7$ $\mathrm{kHz}$ ) and high-frequency ranges, respectively. Results obtained in quiet and noise are shown with black and red axes, respectively. Significance was tested using Student's $t$ tests. $\boldsymbol{M}$, Correlation between neural and behavioral thresholds across frequencies and backgrounds (quiet, black symbols; noise, red symbols) retaining as the neural threshold the lowest ("best") value across rate- and Cl-based thresholds. The solid black line is a linear fit of data $\left(r^{2}=0.88\right)$.

$3.1 \mathrm{~dB}$ and $25.25 \pm 4 \mathrm{~dB}$ difference in quiet and noise, respectively; Fig. 5C, $D, G, H)$.

We then compared rate- and CI-based thresholds (Fig. 5I-L) in apical $(\mathrm{CF}<2.7 \mathrm{kHz})$ and basal $(\mathrm{CF}>2.7 \mathrm{kHz})$ regions of the cochlea. In quiet, CI-based neural threshold provides better estimates of behavioral thresholds in the low-frequency range ( $5.3 \pm$ $4.2 \mathrm{~dB}$ for the $\mathrm{CI}$ against $14 \pm 4.6 \mathrm{~dB}$ for rate; Fig. $5 I)$. The opposite trend was observed in the high-frequency range (9.5 $\mathrm{dB} \pm 2.7 \mathrm{~dB}$ for rate compared with $19.3 \mathrm{~dB} \pm 3.1 \mathrm{~dB}$ for CI; Fig. $5 J)$. In noise, this effect is enhanced (Fig. $5 K, L$ ), attesting to the complementary role of rate and temporal neural coding. To quantify this complementarity, we plotted the best neural threshold determined either through CI or discharge rate as a function of behavioral threshold in the same octave band (Fig. 5M). The distribution of data parallels the $y=x$ line with an offset of $8 \mathrm{~dB}$, demonstrating that spike time and spike rate play complemen- tary roles in the efficient detection of tones in noise even if behavioral thresholds show a better sensitivity and whatever the mode of coding (rate vs time) and the background (quiet vs noise).

\section{Involvement of low- and high-SR fibers in} tone-in-noise detection

In most studies, the low-SR ANFs, which have higher rate-based threshold than high-SR fibers, have been proposed to be the prominent pool operating in noisy backgrounds (Costalupes et al., 1984; Young and Barta, 1986). To address this hypothesis, we plotted the behavioral threshold and the population threshold according to the fiber's SR (Fig. $6 A-D$ ). To determine which population accounts for behavioral thresholds, we then measured the threshold difference between the fiber's SR and those obtained using the acoustical startle reflex below and above 2.7 $\mathrm{kHz}$ (Fig. 6E-H). In quiet, the high-SR fibers, which represent 

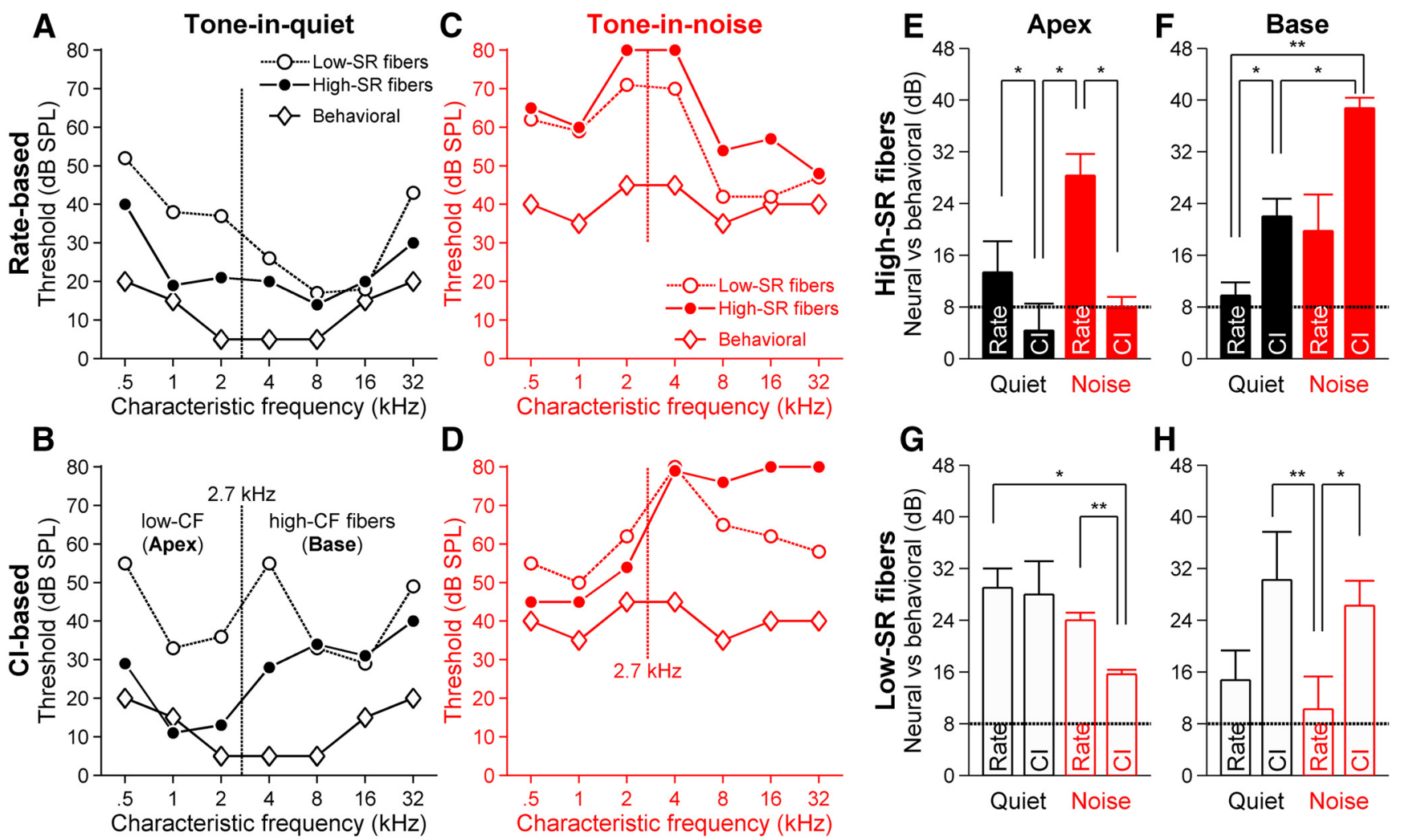

Figure 6. Neural population thresholds in quiet and noise estimated from low- and high-SR ANFs. $\boldsymbol{A}-\boldsymbol{D}$, Rate-based and $\mathrm{Cl}$-based population thresholds in quiet ( $\boldsymbol{A}$, rate; $\boldsymbol{B}$, (I) and noisy conditions ( $\boldsymbol{C}$, rate; $\boldsymbol{D}, \boldsymbol{C l})$ considering separately low-SR fibers ( $<20$ spikes/s, open circles) and high-SR fibers (closed circles). Diamond symbols indicate behavioral auditory thresholds. $\boldsymbol{E}-\boldsymbol{H}$, Quantification of the data shown in $\boldsymbol{A}-\boldsymbol{D}$ by partitioning the ANFs into four pools according to SR and CF as follows: (1) low-CF/high-SR (E, 31\% of the ANFs), (2) high-CF/high-SR ( $\boldsymbol{F}, 27 \%)$, (3) low-CF/low-SR (G,6\%), and high-CF/low-SR fibers $(\boldsymbol{H}, 36 \%)$. Dashed horizontal line at $8 \mathrm{~dB}$ shows the difference between neural and behavioral thresholds estimated in Figure $5 M$. Significance was tested using Student's $t$ test for paired samples. ${ }^{*} p<0.05 ;{ }^{* *} p<0.01$.

$58 \%$ of the whole ANFs, represent behavioral thresholds through rate spiking $>2.7 \mathrm{kHz}$; that is, the basal part of the cochlea containing $27 \%$ of the high-SR ANF $(9.7 \pm 2 \mathrm{~dB}$ threshold difference in quiet; Fig. $6 A, F$ ). Below this frequency, the behavior thresholds are best approximated using the time-based threshold of high-SR fibers (i.e., the apical part of the cochlea containing $31 \%$ of the high-SR ANFs, $4.3 \pm 4.1 \mathrm{~dB}$ threshold difference in quiet; Fig. $6 B, E)$. In noise, time-based thresholds of high-SR fibers are similar to the behavior thresholds at low frequency $(8 \pm 1.5 \mathrm{~dB}$; Fig. $6 D, E)$, whereas low-SR fibers of the basal turn $(36 \%$ of the ANFs) account for behavioral thresholds using the rate domain $(10.2 \pm 5.1 \mathrm{~dB}$; Fig. $6 C, H)$. Altogether, these results suggest that high-SR fibers are the major component in detecting lowfrequency tone-in-noise, whereas high-frequency tones in noise are mostly detected by the low-SR fibers.

\section{Discussion}

In this study, we took advantage of the SAC and its derived CI (Joris et al., 2006) as a unique proxy to quantify the spike synchrony in the whole ANF population (i.e., from $0.4-40 \mathrm{kHz}$ ). This method makes it possible to measure neural phase locking at low frequencies and the first spike jitter in the high frequencies independently of the tone frequency and thus determine the individual single-unit threshold. By comparing the neural population thresholds and behavioral thresholds, we demonstrate that low-frequency/high-SR fibers detect tone-in-noise through temporal coding even if the rate intensity function shows no noticeable increment, whereas the high-frequency/low-SR fibers rely on rate coding. Altogether, our results emphasize the comple- mentarity of spike-time and spike-rate codes in the detection of tone-in-noise.

\section{Sound-encoding mechanisms in noise}

It is generally accepted that high-SR ANFs populating the apical part of the cochlea are more adapted to encode temporal information because the large AC receptor potential from the IHCs can modulate the firing rate above and below the SR of the fibers (Patuzzi, 2011). In our study, low-frequency/high-SR ANFs have better time-based thresholds than rate-based (as attested by negative $\Delta$ threshold), which is consistent with a rearrangement of spike timing before an elevation of the discharge rate (Anderson et al., 1971; Johnson, 1980; Köppl, 1997). It is thus not surprising to find a majority of high-SR fibers at the apex $(\mathrm{CF}<2.7 \mathrm{kHz})$ of the gerbil cochlea (Huet et al., 2016). In contrast, low-frequency/ low-SR ANFs first increase their discharge rate before reorganize their spiking time and then rely more on the rate domain rather than the time domain to code threshold. As shown by the positive value of the $\Delta$ threshold, detection of high-frequency tones $(>2.7$ $\mathrm{kHz}$ ) by both high- and low-SR ANFs always depends on the increment in the spike rate preceding a noticeable synchronization (Rhode and Smith, 1985).

Adding a continuous broadband noise increases the basal firing rate of the fibers (busy-line masking), decreases the maximum discharge rate (adapting masking), and shifts the dynamic range of the rate intensity function to higher sound pressure levels (suppression masking) (Delgutte, 1996; Heil and Peterson, 2015). The spread of excitation produced by the masker to the place of the tone along the cochlea activates the sensory hair cell 
to drive glutamate release onto the fibers (busy-line). When the duration of a noise masker is sufficiently long to adapt the fiber firing (adapting masking), the response to a subsequent tone burst is reduced because presynaptic vesicles from the readily releasable pool are depleted, resulting in a reduction of the maximal sound-evoked discharge rate (Moser and Beutner, 2000; Beutner et al., 2001; Spassova et al., 2004; Goutman and Glowatzki, 2007; Frank et al., 2010; Heil and Peterson, 2015). Although busy-line masking and adapting masking compress the rate intensity function, they are unlikely to change the fiber's threshold in a significant manner. Therefore, a change in the mechanical response of the basilar membrane that has been demonstrated using two-tone paradigms (Sachs and Kiang, 1968; Abbas, 1978; Javel et al., 1978; Delgutte, 1990; Ruggero et al., 1992) is more likely to account for elevated masking thresholds (RecioSpinoso and Cooper, 2013). Finally, other adaptation mechanisms such as dynamic-range adaptation to sound level statistics may also participate in the modulation of rate intensity functions (Wen et al., 2009).

In tone-in-noise, we showed that low-frequency/high-SR ANFs continue to use spike time to encode threshold independently of their maximum discharge rate. Indeed, fibers with no detectable rate-based threshold fire action potentials in synchrony with the fine structure of the tone in noise (our study). The large fraction of this ANFs pool (i.e., 31\% of the whole contingent; Fig. $6 E$ ) makes temporal coding the major mechanism in detecting low-frequency tones in noise. Moreover, the small fraction of apical low-SR fibers with low CF (6\%; Fig. 6G) switch their mode of threshold encoding from rate-based in quiet to timebased in noise (e.g., Fig. 2Q,R). Beyond the neural phase-locking range, all the ANFs use spike rate to encode thresholds (Fig. $6 F, H)$. It is generally accepted that high- and low-SR ANFs are distributed on the pillar and the modiolus side of the IHCs, respectively (Liberman, 1982). Modiolus synapse properties potentially confer on low-SR fibers this higher robustness in noise. They have a larger active zone and more $\mathrm{Ca}^{2+}$ channels that operate in a more depolarized range (Ohn et al., 2016), leading to a higher fiber activation threshold. Alternatively, the resistance to noise may be due to the efferent loops that modulate auditory nerve signaling in response to sound (Guinan et al., 2012). The medial olivocochlear efferent fibers may provide an antimasking effect by reducing outer hair cell gain, which in turn would reduce the firing of the ANFs to sustained noise and increased sensitivity to tones (Kawase et al., 1993). The lateral efferent pathway, which acts directly upon the synapse between the IHCs and the ANFs with a stronger efferent innervation on the modiolar side, could give low-SR fibers an advantage in encoding sound in the presence of background noise (Guinan, 2006; Guinan et al., 2012).

\section{Complementary spike-time and spike-rate coding for behavioral thresholds}

Auditory brainstem responses and behavioral conditioning paradigm are commonly used for measuring hearing thresholds in animals and humans. Here, we used the prepulse modulation of the acoustical startle reflex. The startle reflex is driven by cochlear nerve input to the cochlear root neurons, which project to the sensorimotor interface in the caudal pontine reticular nucleus, and then excites spinal motor neurons responsible for the wholebody startle response (Lingenhöhl and Friauf, 1994; Lee et al., 1996). Prepulse inhibition has been used successfully to measure the auditory threshold objectively in quiet and in noisy backgrounds (Hickox and Liberman, 2014), as well as to identify behavioral correlates of cochlear damage due to ototoxic drugs
(Young and Fechter, 1983; Chambers et al., 2016) and of temporary threshold shifts due to pure tone acoustic exposure (Walter et al., 2012). Advantages of prepulse modulation of the acoustical startle reflex are as follows: (1) it can be collected in awake animals over a large frequency range, especially the low-frequency tones that cannot be explored by the auditory brainstem responses, because of the neural phase-locking; (2) it does not require weeks or months of training, as in other commonly used behavioral paradigms; and (3) many animals can be tested at once with a short preparation, allowing for high data throughput and timely data collections. Moreover, the strength of the present study relies on careful correlations between behavioral thresholds obtained from a large number of animals $(n=41$ gerbils) and ANF population thresholds from a large ANF data collection ( $n=337$ ANFs in 39 gerbils), according time- and rate-based modes and their SR for tone-in-noise detection in an animal model with sensitivity to low-frequency sounds.

Here, we report that behavioral thresholds correlate with the high-SR fiber threshold based on the time domain in the phaselocking range $(<2.7 \mathrm{kHz})$ and the rate domain at higher frequencies. Although high-SR fibers use temporal coding for detecting tone-in-noise in the phase-locking range, another pool of ANFs, the low-SR fibers, engage in threshold coding using the rate domain at higher frequencies. The interplay between the two pools enables the detection of tone-in-noise along the entire tonotopic axis. Between 2 and $4 \mathrm{kHz}$, the tone-in-noise behavioral audiogram shows a notch, which may correspond to the transition zone between the two modes of encoding; that is, a temporal encoding mode with a prevalence of high-SR (31\%) versus low-SR (6\%) ANFs in the apical part of the cochlea and a rateencoding mode in the basal part with a more balanced distribution of high-SR (27\%) and low-SR (36\%) fibers. Moreover, we report that the behavioral thresholds were always more sensitive than the fiber thresholds by $8 \mathrm{~dB}$ (Fig. $5 \mathrm{M}$ ).

This offset in favor of behavioral thresholds may result from the small proportion of the fibers recorded ( $\sim 2 \%$ of the whole ANFs), the possibility that behavioral threshold are coded by the lowest threshold fibers (Delgutte, 1996), the anesthesia procedures, which may affect the efferent olivocochlear bundle and thus the single-fiber properties (Winslow and Sachs, 1988), or the integration of the information into the ventral and dorsal cochlear nuclei, which have been shown to contribute to the startle response (for review, see Yeomans and Frankland, 1995; Meloni and Davis, 1998). In addition, we performed single-unit recording in one ear, contrasting to the prepulse inhibition of the acoustical startle reflex, in which two ears are involved for tone detection (Hoffman and Ison, 1980; Fendt et al., 2001). Finally, the statistical method used in this study to compute the population thresholds (i.e., one-way ANOVA) may overestimate the neural threshold of few decibels compared with others approaches that take into account the most sensitive fibers (Winslow and Sachs, 1988). Further studies that integrate the central projection of ANFs on cochlear nuclei and apply a signal detection theory with computational neural models may provide another optimal estimate of neural thresholds (Heinz et al., 2001).

To summarize, we have demonstrated that low-CF/high-SR and high-CF/low-SR ANFs using, respectively, spiking-time and spiking-rate modes to encode threshold are particularly adapted to detect tones in noise, and thus have a complementary role to encode behavioral threshold. Because of the frequency range of hearing in gerbils $(\sim 0.4-40 \mathrm{kHz})$, it can be hypothesized that similar mechanisms operate in human hearing during the detection of tones in a noisy environment. 


\section{References}

Abbas PJ (1978) Effects of stimulus frequency on two-tone suppression: a comparison of physiological and psychophysical results. J Acoust Soc Am 63:1878-1886. CrossRef Medline

Abbas PJ (1981) Auditory-nerve fiber responses to tones in a noise masker. Hear Res 5:69-80. CrossRef Medline

Anderson DJ, Rose JE, Hind JE, Brugge JF (1971) Temporal position of discharges in single auditory nerve fibers within the cycle of a sine-wave stimulus: frequency and intensity effects. J Acoust Soc Am 49:1131+. Medline

Beutner D, Voets T, Neher E, Moser T (2001) Calcium dependence of exocytosis and endocytosis at the cochlear inner hair cell afferent synapse. Neuron 29:681-690. CrossRef Medline

Bourien J, Tang Y, Batrel C, Huet A, Lenoir M, Ladrech S, Desmadryl G, Nouvian R, Puel JL, Wang J (2014) Contribution of auditory nerve fibers to compound action potential of the auditory nerve. J Neurophysiol 112:1025-1039. CrossRef Medline

Chambers AR, Resnik J, Yuan Y, Whitton JP, Edge AS, Liberman MC, Polley DB (2016) Central gain restores auditory processing following nearcomplete cochlear denervation. Neuron 89:867-879. CrossRef Medline

Costalupes JA (1983) Broadband masking noise and behavioral pure tone thresholds in cats. J Acoust Soc Am 74:758-764. CrossRef Medline

Costalupes JA (1985) Representation of tones in noise in the responses of auditory nerve fibers in cats. I. Comparison with detection thresholds. J Neurosci 5:3261-3269. CrossRef Medline

Costalupes JA, Young ED, Gibson DJ (1984) Effects of continuous noise backgrounds on rate response of auditory nerve fibers in cat. J Neurophysiol 51:1326-1344. CrossRef Medline

Davis M (1974) Sensitization of the rat startle response by noise. J Comp Physiol Psychol 87:571-581. CrossRef Medline

Delgutte B (1990) Two-tone rate suppression in auditory-nerve fibers: dependence on suppressor frequency and level. Hear Res 49:225-246. CrossRef Medline

Delgutte B (1996) Physiological models for basic auditory percepts. In: Auditory computation (Hawkins HL, McMullen TA, Popper AN, Fay RR, eds), pp 157-220. New York, NY: Springer.

Fendt M, Li L, Yeomans JS (2001) Brain stem circuits mediating prepulse inhibition of the startle reflex. Psychopharmacology (Berl) 156:216-224. CrossRef Medline

Frank T, Rutherford MA, Strenzke N, Neef A, Pangršič T, Khimich D, Fejtova A, Fetjova A, Gundelfinger ED, Liberman MC, Harke B, Bryan KE, Lee A, Egner A, Riedel D, Moser T (2010) Bassoon and the synaptic ribbon organize $\mathrm{Ca}^{2+}$ channels and vesicles to add release sites and promote refilling. Neuron 68:724-738. CrossRef Medline

Goutman JD, Glowatzki E (2007) Time course and calcium dependence of transmitter release at a single ribbon synapse. Proc Natl Acad Sci U S A 104:16341-16346. CrossRef Medline

Guinan JJ Jr (2006) Olivocochlear efferents: anatomy, physiology, function, and the measurement of efferent effects in humans. Ear Hear 27:589-607. CrossRef Medline

Guinan JJ Jr, Salt A, Cheatham MA (2012) Progress in cochlear physiology after Békésy. Hear Res 293:12-20. CrossRef Medline

Heil P, Peterson AJ (2015) Basic response properties of auditory nerve fibers: a review. Cell Tissue Res 361:129-158. CrossRef Medline

Heinz MG, Colburn HS, Carney LH (2001) Evaluating auditory performance limits: i. one-parameter discrimination using a computational model for the auditory nerve. Neural Comput 13:2273-2316. CrossRef Medline

Henry KS, Heinz MG (2012) Diminished temporal coding with sensorineural hearing loss emerges in background noise. Nat Neurosci 15:13621364. CrossRef Medline

Hickox AE, Liberman MC (2014) Is noise-induced cochlear neuropathy key to the generation of hyperacusis or tinnitus? J Neurophysiol 111:552-564. CrossRef Medline

Hoffman HS, Ison JR (1980) Reflex modification in the domain of startle: I. some empirical findings and their implications for how the nervous system processes sensory input. Psychol Rev 87:175-189. CrossRef Medline

Huet A, Batrel C, Tang Y, Desmadryl G, Wang J, Puel JL, Bourien J (2016) Sound coding in the auditory nerve of gerbils. Hear Res 338:32-39. CrossRef Medline

Javel E, Geisler CD, Ravindran A (1978) Two-tone suppression in auditory nerve of the cat: rate-intensity and temporal analyses. J Acoust Soc Am 63:1093-1104. CrossRef Medline

Johnson DH (1980) The relationship between spike rate and synchrony in responses of auditory-nerve fibers to single tones. J Acoust Soc Am 68: 1115-1122. CrossRef Medline

Joris PX, Louage DH, Cardoen L, van der Heijden M (2006) Correlation index: a new metric to quantify temporal coding. Hear Res 216-217: 19-30. CrossRef Medline

Kawase T, Delgutte B, Liberman MC (1993) Antimasking effects of the olivocochlear reflex. II. Enhancement of auditory-nerve response to masked tones. J Neurophysiol 70:2533-2549. CrossRef Medline

Kittel M, Wagner E, Klump GM (2002) An estimate of the auditory-filter bandwidth in the Mongolian gerbil. Hear Res 164:69-76. CrossRef Medline

Köppl C (1997) Phase locking to high frequencies in the auditory nerve and cochlear nucleus magnocellularis of the barn owl, Tyto alba. J Neurosci 17:3312-3321. CrossRef Medline

Kortlang S, Mauermann M, Ewert SD (2016) Suprathreshold auditory processing deficits in noise: effects of hearing loss and age. Hear Res 331:2740. CrossRef Medline

Lee Y, López DE, Meloni EG, Davis M (1996) A primary acoustic startle pathway: obligatory role of cochlear root neurons and the nucleus reticularis pontis caudalis. J Neurosci 16:3775-3789. CrossRef Medline

Liberman MC (1982) The cochlear frequency map for the cat: labeling auditory-nerve fibers of known characteristic frequency. J Acoust Soc Am 72:1441-1449. CrossRef Medline

Lingenhöhl K, Friauf E (1994) Giant neurons in the rat reticular formation: a sensorimotor interface in the elementary acoustic startle circuit? J Neurosci 14:1176-1194. CrossRef Medline

Lorenzi C, Gilbert G, Carn H, Garnier S, Moore BC (2006) Speech perception problems of the hearing impaired reflect inability to use temporal fine structure. Proc Natl Acad Sci U S A 103:18866-18869. CrossRef Medline

Louage DH, van der Heijden M, Joris PX (2004) Temporal properties of responses to broadband noise in the auditory nerve. J Neurophysiol 91: 2051-2065. CrossRef Medline

Macmillan NA, Creelman CD (2004) Detection theory: a user's guide, Ed 2. Mahwah, NJ: Lawrence Erlbaum.

Meloni EG, Davis M (1998) The dorsal cochlear nucleus contributes to a high intensity component of the acoustic startle reflex in rats. Hear Res 119:69-80. CrossRef Medline

Moore BC, Raab DH (1975) Intensity discrimination for noise bursts in the presence of a continuous, bandstop background: effects of level, width of the bandstop, and duration. J Acoust Soc Am 57:400-405. CrossRef Medline

Moore BCJ (2003) An introduction to the psychology of hearing, Ed 5. Boston, MA: Academic.

Moser T, Beutner D (2000) Kinetics of exocytosis and endocytosis at the cochlear inner hair cell afferent synapse of the mouse. Proc Natl Acad Sci U S A 97:883-888. CrossRef Medline

Ohn TL, Rutherford MA, Jing Z, Jung S, Duque-Afonso CJ, Hoch G, Picher MM, Scharinger A, Strenzke N, Moser T (2016) Hair cells use active zones with different voltage dependence of $\mathrm{Ca}^{2+}$ influx to decompose sounds into complementary neural codes. Proc Natl Acad Sci U S A 113: E4716-E4725. CrossRef Medline

Palmer AR, Russell IJ (1986) Phase-locking in the cochlear nerve of the guinea-pig and its relation to the receptor potential of inner hair-cells. Hear Res 24:1-15. CrossRef Medline

Patuzzi R (2011) Ion flow in cochlear hair cells and the regulation of hearing sensitivity. Hear Res 280:3-20. CrossRef Medline

Recio-Spinoso A, Cooper NP (2013) Masking of sounds by a background noise-cochlear mechanical correlates. J Physiol 591:2705-2721. CrossRef Medline

Rhode WS, Smith PH (1985) Characteristics of tone-pip response patterns in relationship to spontaneous rate in cat auditory nerve fibers. Hear Res 18:159-168. CrossRef Medline

Rhode WS, Geisler CD, Kennedy DT (1978) Auditory nerve fiber response to wide-band noise and tone combinations. J Neurophysiol 41:692-704. CrossRef Medline

Rose JE, Brugge JF, Anderson DJ, Hind JE (1967) Phase-locked response to low-frequency tones in single auditory nerve fibers of the squirrel monkey. J Neurophysiol 30:769-793. CrossRef Medline 
Ruggero MA, Robles L, Rich NC (1992) Two-tone suppression in the basilar membrane of the cochlea: mechanical basis of auditory-nerve rate suppression. J Neurophysiol 68:1087-1099. CrossRef Medline

Ryan A (1976) Hearing sensitivity of the Mongolian gerbil, Meriones unguiculatis. J Acoust Soc Am 59:1222-1226. CrossRef Medline

Sachs MB, Kiang NY (1968) Two-tone inhibition in auditory-nerve fibers. J Acoust Soc Am 43:1120-1128. CrossRef Medline

Spassova MA, Avissar M, Furman AC, Crumling MA, Saunders JC, Parsons TD (2004) Evidence that rapid vesicle replenishment of the synaptic ribbon mediates recovery from short-term adaptation at the hair cell afferent synapse. J Assoc Res Otolaryngol 5:376-390. CrossRef Medline

Versteegh CP, Meenderink SW, van der Heijden M (2011) Response characteristics in the apex of the gerbil cochlea studied through auditory nerve recordings. J Assoc Res Otolaryngol 12:301-316. CrossRef Medline

Vinay, Moore BC (2007) Ten(HL)-test results and psychophysical tuning curves for subjects with auditory neuropathy. Int J Audiol 46:39-46. CrossRef Medline

Walter M, Tziridis K, Ahlf S, Schulze H (2012) Context dependent auditory thresholds determined by brainstem audiometry and prepulse inhibition in Mongolian gerbils. Open J Acoust 2:34-49. CrossRef
Weiss TF, Rose C (1988) A comparison of synchronization filters in different auditory receptor organs. Hear Res 33:175-179. CrossRef Medline

Wen B, Wang GI, Dean I, Delgutte B (2009) Dynamic range adaptation to sound level statistics in the auditory nerve. J Neurosci 29:13797-13808. CrossRef Medline

Winslow RL, Sachs MB (1988) Single-tone intensity discrimination based on auditory-nerve rate responses in backgrounds of quiet, noise, and with stimulation of the crossed olivocochlear bundle. Hear Res 35:165-189. CrossRef Medline

Yeomans JS, Frankland PW (1995) The acoustic startle reflex: neurons and connections. Brain Res Brain Res Rev 21:301-314. CrossRef Medline

Young ED, Barta PE (1986) Rate responses of auditory nerve fibers to tones in noise near masked threshold. J Acoust Soc Am 79:426-442. CrossRef Medline

Young JS, Fechter LD (1983) Reflex inhibition procedures for animal audiometry: a technique for assessing ototoxicity. J Acoust Soc Am 73:16861693. CrossRef Medline

Zeng FG, Kong YY, Michalewski HJ, Starr A (2005) Perceptual consequences of disrupted auditory nerve activity. J Neurophysiol 93:30503063. CrossRef Medline 